\title{
Bilateral adrenal masses
}

\author{
E Lopez, G Piédrola, L Villalon
}

\section{Case report}

A 68-year-old woman presented with pain in the left hypochondrium and lumbar fossa, nausea, and constitutional symptoms during a two-month period. Physiological examination revealed a painful mass with irregular edges in that area without adenopathies or organomegalies.

\section{INVESTIGATIONS}

Analyses on admission were normal except an erythrocyte sedimentation rate of 42 in the first hour, $\mathrm{Na} 126 \mathrm{mEq} / 1, \mathrm{~K} 6.2 \mathrm{mEq} / 1$, and lactate dehydrogenase $1446 \mathrm{U} / 1$. The basal hormonal study (catecholamines, metanephrines, testosterone, DHEA-S, plasmatic renin activity, aldosterone, adrenocorticotropic hormone (ACTH) and urinary free cortisol) was also normal. Cosyntropin stimulation test (250 $\mu \mathrm{g}$ of synthetic 1-24 ACTH IV) showed serum cortisol values of $22.4,22.2$ and $23.3 \mu \mathrm{g} / \mathrm{dl}$ at 0,30 and 60 minutes, respectively.
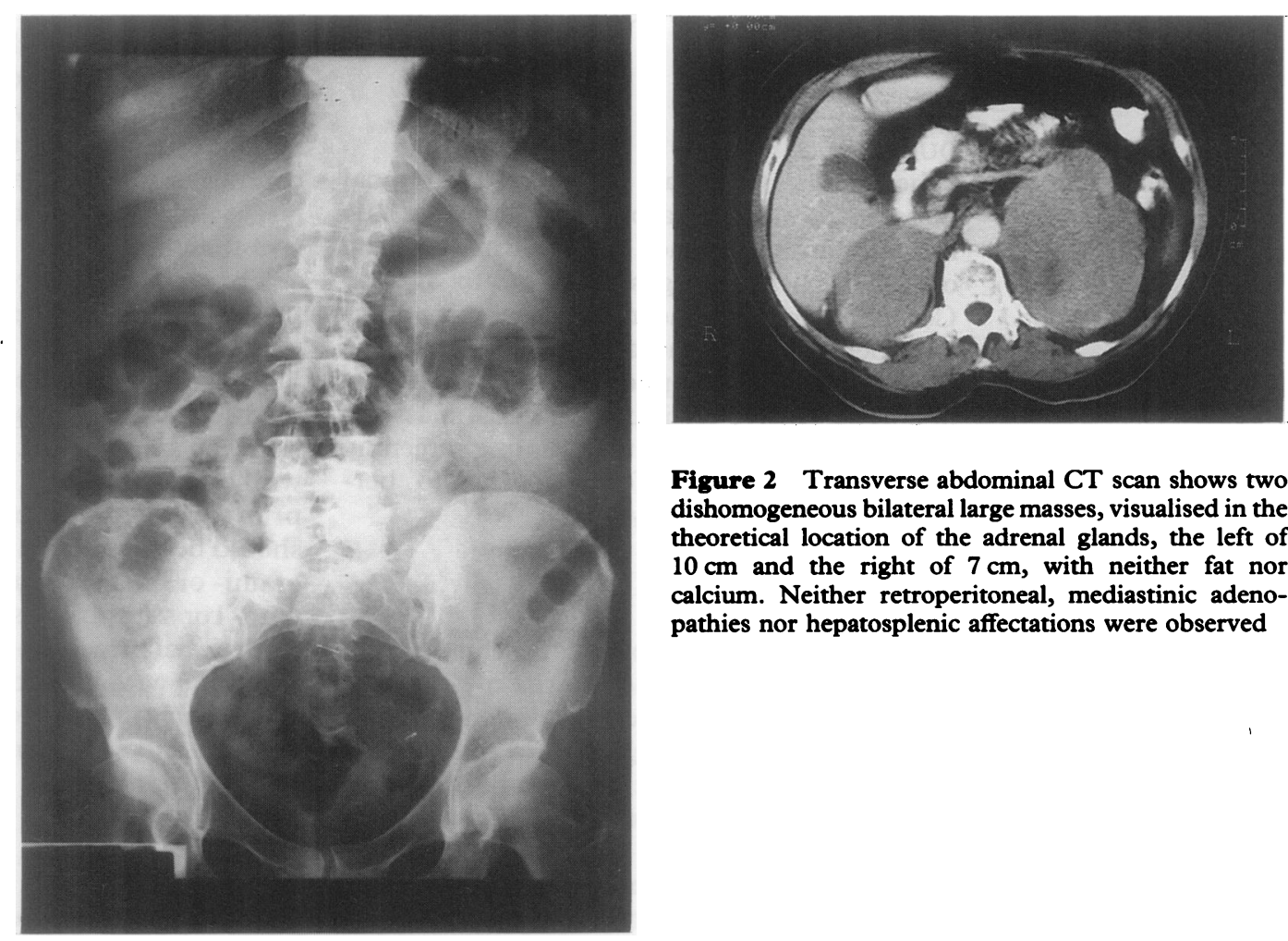

Figure 2 Transverse abdominal CT scan shows two dishomogeneous bilateral large masses, visualised in the theoretical location of the adrenal glands, the left of $10 \mathrm{~cm}$ and the right of $7 \mathrm{~cm}$, with neither fat nor calcium. Neither retroperitoneal, mediastinic adenopathies nor hepatosplenic affectations were observed

\section{Departments of Endocrinology and Haematology, Ramon y Cajal Hospital, 28034 Madrid, Spain E Lopez \\ G Piédrola \\ L Villalon}

Accepted 2 March 1995
Figure 1 Abdominal radiography. The left kidney is displaced downward and outward, without evidence of abdominal masses

\section{Questions}

1 What is your differential diagnosis?

2 What diagnostic test would you perform?

3 Has this patient an adrenal insufficiency? 


\section{Answers}

\section{QUESTION 1}

The most important causes of bilateral adrenal masses are represented in the box. Phaeochromocytoma and myelolipoma were excluded in this patient because catecholamine values were normal and there was no fat on computed tomography (CT). Bilateral adrenal haemorrhages are rare without anticoagulation or previous trauma. ${ }^{1}$ Uni- or bilateral tuberculosis abscesses are infrequent and the presence of calcium is usual. ${ }^{1}$ Our principal diagnostic suspicion in this patient, with bilateral adrenal masses and constitutional symptoms, was metastasis from a distant malignancy. Adrenal glands are a common site of metastasic deposits from breast, gastric, renal, pancreatic, colon and lung carcinomas, melanomas, and malignant lymphomas, usually non-Hodgkin's lymphomas. ${ }^{2}$ Another diagnostic possibility was adrenocortical carcinoma, which is bilateral in up to $10 \% ;$ it is usually large at presentation with abdominal pain or palpable mass. ${ }^{3}$ Half of these tumours are functional and may be detected by manifestations of excess hormone production, which were not present in our patient. $^{3}$

\section{QUESTION 2}

When metastasic disease is suspected in adrenal mass, percutaneous aspiration biopsy is the most definitive method of confirmation; the overall accuracy ranges from 80 to $100 \%$. $^{3}$ Complications are uncommon ${ }^{3}$ the most common being pneumothorax, usually small. The most worrisome complication is precipitation of a hypertensive crisis if the adrenal mass is a phaeochromocytoma; catecholamine screening should always be performed prior to percutaneous needle biopsy. ${ }^{2,3}$

\section{QUESTION 3}

The patient had a normal baseline serum cortisol level, which failed to rise after cosyntropin stimulation, documenting a lack of adrenal reserve, although she did not have an absolute adrenal insufficiency, as peak cortisol level exceeded $18 \mu \mathrm{g} / \mathrm{dl}^{4}{ }^{4}$ Clinical adrenal insufficiency in patients with invasion of these glands by metastasis or other pathologies is not common, since for this to occur, at least $90 \%$ of the glandular tissue must be destroyed ${ }^{5}$; loss of adrenal reserve is more frequent and has been described in patients with bilateral adrenal metastasis. $^{5}$

1 Billaud K, Benabed E, Requeda E, et al. Lésions tumorales and pseudo-tumorales de la loge surrénalienne à ne pas and pseudo-tumorales de la loge surrénal

2 Berkman WA, Bernardino ME, Sewell CW, et al. The computed tomography-guided adrenal biopsy. An alternative computed tomography-guided adrenal biopsy. An alternative
to surgery in adrenal mass diagnosis. Cancer 1984; 53:
$2098-103$.

3 Dunnick NR. Adrenal imaging: current status. $A \mathcal{F} 1990$ 154: 927-36.
Causes of bilateral adrenal masses

- metastasis of a distant malignancy

- bilateral adrenal phaeochromocytoma

- bilateral adrenal lymphoma (primary or secondary)

- bilateral adrenal myelolipoma

- bilateral adrenocortical carcinoma

- bilateral adrenal haemorrhages

- infections (tuberculosis, abscess, etc)

\section{Discussion}

CT-guided fine needle aspiration of the left adrenal revealed medium sized cells of a lymphoid appearance and malignant characteristics, immunoreactive with the leucocyte common antigen, confirming a non-Hodgkin's lymphoma CD45+. Other explorations (thoracic scan, barium enema, gastrointestinal transit, mammography, bone scintigraphy and study of the bone marrow) were normal. Faced with suspected adrenal insufficiency, the patient was treated from the beginning with hydrocortisone which induced normalisation of hydroelectrolytic changes and clinical improvement. During the diagnostic study, however, the patient's overall condition progressively deteriorated with a number of metabolic complications associated with the tumoural lysis syndrome and she died in spite of intensive therapy.

This is a rare case of non-Hodgkin's lymphoma limited to the adrenal glands without evidence of affect elsewhere and with diminished adrenal reserve shown by inadequate cortisol response to cosyntropin stimulation and clinical and analytical improvement with glucocorticoid treatment. Although primary adrenal lymphoma is very rare ${ }^{6}$ it should be included in the differential diagnosis of uni- or bilateral adrenal masses, particularly with constitutional symptomatology and no evidence of primary neoplasm elsewhere. Early diagnosis with percutaneous aspiration biopsy is necessary in order to begin therapy as soon as possible and avoid serious and potentially fatal complications.

\section{Final diagnosis}

Primary bilateral adrenal non-Hodgkin's lymphoma.

Keywords: non-Hodgkin's lymphoma, bilateral adrenal masses

4 Grinspoon SK, Biller BMK. Laboratory assessment of adrenal insufficiency. F Clin Endocrinol Metab 1994; 79: 923-31

5 Redman BG, Pazdur R, Zingas A, et al. Prospective evaluation of adrenal insufficiency in patients with adrenal metastasis. Cancer 1987; 60: 103-7.

6 Harris GJ, Tio FO, Von Hoff DD. Primary adrenal lymphoma. Cancer 1989; 63: 799-803. 